\title{
Finite Element Analysis of Small Span Reinforced Concrete Trough Railway Bridge
}

\author{
Zhongquan Zou ${ }^{1,2, \mathrm{a}}$, Ola Enochsson ${ }^{2, \mathrm{~b}}$, Guojing $\mathrm{He}^{3, \mathrm{c}}$ and Lennart Elfgren ${ }^{2, \mathrm{~d}}$ \\ 1. College of Civil Engineering and Architecture, Central South University, \\ Changsha, China, 410075; \\ 2. Div. of Structural Engineering, Dept. of Civil \& Environmental Engineering, \\ Luleå University of Technology, Luleå, Sweden, SE-97187; \\ 3. College of Civil Engineering, Architecture and Mechanics, The Central South \\ University of Forestry and Technology, Changsha, China, 410075

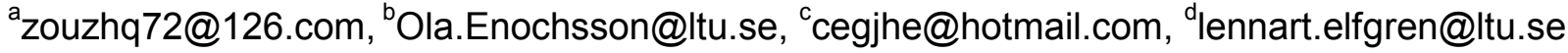

\begin{abstract}
Keywords: Reinforced concrete, trough railway bridge, finite element analysis, load bearing capacity assessment
\end{abstract}

\begin{abstract}
The static behavior of a reinforced concrete trough railway bridge is analyzed by finite element method. The influences of load distribution fashion, supporting width of the bearings and the additional support beneath the girder are investigated; and the spatial effect of the stress distribution is studied as well. To confirm the analysis accuracy, the results are calibrated with the field load test results. It is found that the influence of load distribution fashion is minor and negligible, while those of supporting width and additional support are not negligible; and the spatial effect is significant.
\end{abstract}

\section{Introduction}

The Luossajokk Bridge (Fig. 1) is a reinforced concrete railway bridge, located in Kiruna, Sweden. The Swedish Railway Administrator "Banverket" plans to upgrade the load class of the line from an axle load of 25 tons to an axle load of 30 tons, so its load bearing capacity are required to be assessed.
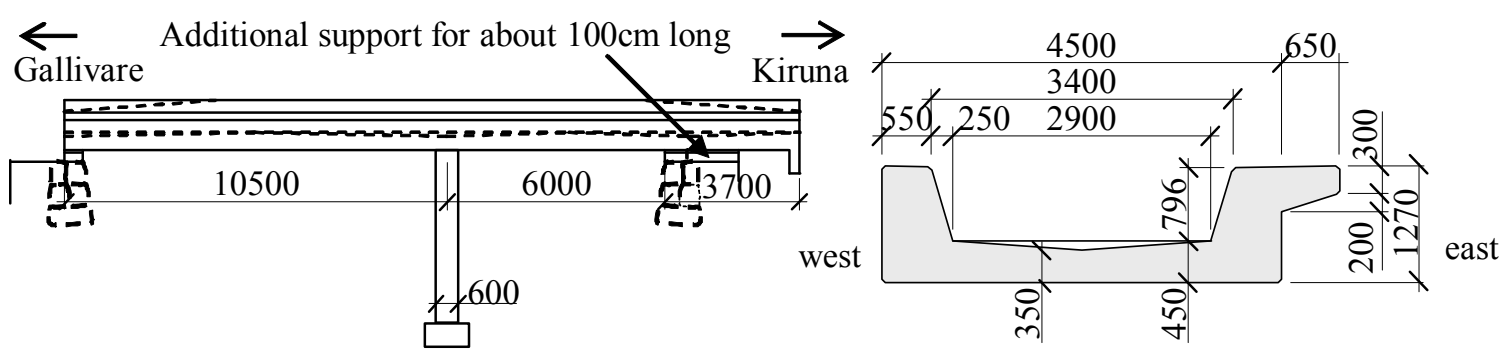

a) Elevation view

b) Cross-section

Fig. 1 The elevation view and cross-section of the bridge [unit: $\mathrm{mm}$ ]

The most particular feature with the Luossajokk Bridge is that both of the two spans are not very long, so that the structure exhibits some peculiar mechanical behavior. First of all, for such a small-span continuous structure with the width of $5.15 \mathrm{~m}$, which is comparable with the length of the span, there will inevitably be some spatial effect on the distribution of the stresses and displacements in the structure, which is not as prominent in a usual-span beam bridge. Further more, as shown in Fig. 1, there is an additional support for about $100 \mathrm{~cm}$ long beneath the east side of the cantilever part of the girder and close to the bearing. The additional support may yield even sharper spatial effect in the structure. Second, as the length of either span is not very long compared with the bearing width, and the bearing is merely a rigid concrete block, which can introduce some uncertainty with the exact actual supporting position, the width of the bearing may also bring some influence on the mechanical 
behavior of the bridge. Therefore, in order to learn the static behavior of the Luossajokk Bridge, and to achieve more precise load bearing capacity assessment, theoretic calculations are performed by finite element method. By making use of the software of ABAQUS 6.7-1, several FE-models have been developed to investigate the following items: i) the effect of support width on the static behavior of the bridge; ii) the effect of the additional support beneath the east side of the beam on the stress distribution; iii) the spatial distribution details of the stresses and displacements in the girder. Finally, based on the analysis results, some suggestions on the load bearing capacity assessment are proposed.

\section{Modeling Details}

Material and Geometric Properties. The material of Luossajokk Bridge is concrete K400 (Specified in the Swedish code BBK(94) ), for which the material properties can be adopted as listed in Table 1.

Table 1 Material properties of K400

\begin{tabular}{cccc}
\hline Item & Elastic modulus $[\mathrm{GPa}]$ & Mass density $\left[\mathrm{t} / \mathrm{m}^{3}\right]$ & Poisson's ratio \\
\hline Value & 33 & 2.5 & 0.167 \\
\hline
\end{tabular}

For convenience of modeling, the actual cross-section of the girder is simplified as shown in Fig. 2 . The error percent of the area, bending inertia about the horizontal central axis and bending inertia about vertical central axis by the simplification are $0.3 \%, 4.7 \%, 0.4 \%$, respectively. The precision is acceptable in engineering concern.

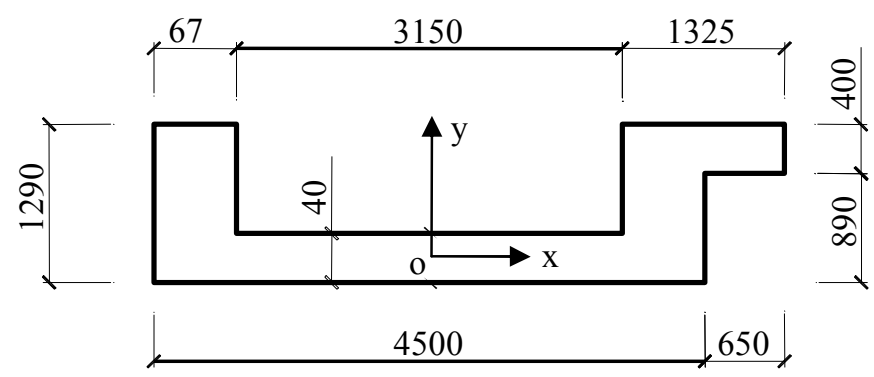

Fig. 2 Simplified cross-section adopted in the FE-analysis [mm]

Analysis Load Case. For convenience and to simplify the analysis, only one load case, i.e., the worst load case for the middle section of the short span, is calculated, and the loads applied in the analysis are in accordance with the actual train axle loads used in the field test, as shown in Fig. 3. It can be found in the figure that the train axle loads are the same in these two positions.

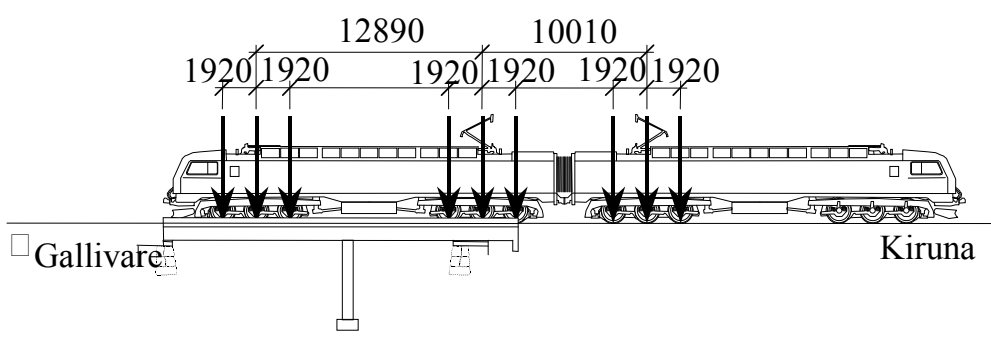

a) Position 1

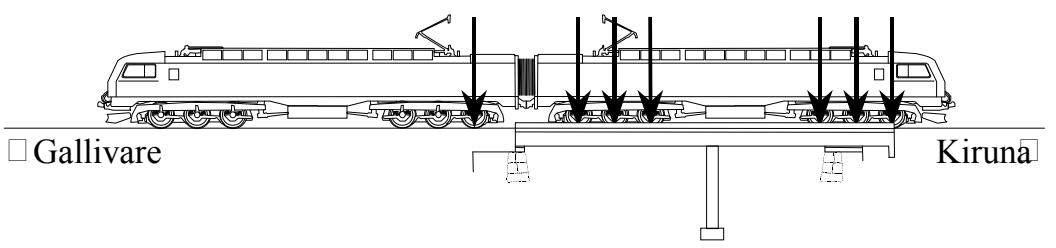

b) Position 2

Fig. 3 Load case applied in the analysis (the figures are excerpted from [1]) 
Finite Element Models. Based on the above considerations and details, several finite element models are developed to investigate the detailed static behavior. To investigate the spatial distribution of the stress and displacement in the bridge, three-dimensional FE-models are developed using shell elements. Yet for comparison purpose and calibration of the three-dimensional models, two-dimensional models using beam elements are also developed. The details of each model are illustrated in Table 2.

Table 2 The details of each FE model

\begin{tabular}{lll}
\hline Model name & Element type & Description \\
\hline Beam_a & Beam elements & $\begin{array}{l}\text { Each supported position is considered to be a simple point, the column is } \\
\text { modeled in a solo straight line }\end{array}$ \\
\hline Beam_b & Beam elements & $\begin{array}{l}\text { Considering the effect of support width by changing the support position, } \\
\text { the column is modeled in double straight lines within its width range }\end{array}$ \\
\hline Shell_a & Shell elements & $\begin{array}{l}\text { Each supported position is considered to be a simple point, the column is } \\
\text { modeled in a solo column }\end{array}$ \\
\hline Shell_b & Shell elements & Considering the additional support based on Shell_a \\
\hline Shell_d & Shell elements & $\begin{array}{l}\text { Considering the effect of support width by changing the support position, } \\
\text { the column is modeled in doble columns within its width range }\end{array}$ \\
\hline
\end{tabular}

\section{Analysis Results}

Influence of Support Width. It is shown in Fig. 4 how the supporting width affect the loading response. When the bridge is not loaded, the actual supporting position can be considered in the middle of the bearing (Fig. 4(a)); yet while it is loaded, the actual supporting position will move according to the deflection line (Fig. 4(b)). The extreme situation is that the supporting position can be moved to one edge of the bearing, as shown in Fig. 4(b). Therefore beam_a, shell_a and shell_b are developed with the normal supporting position as shown in Fig. 4(a), while in beam_b, shell_c and shell_d, the actual supporting position is supposed to be related to the deflection shape of the girder and the influence of column width is considered by modeling it with double columns, as shown in Fig. 4(b).

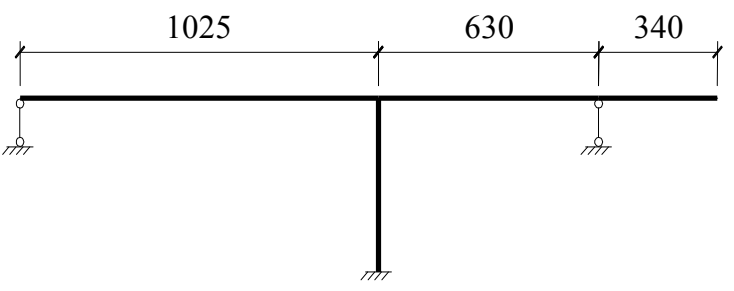

(a)

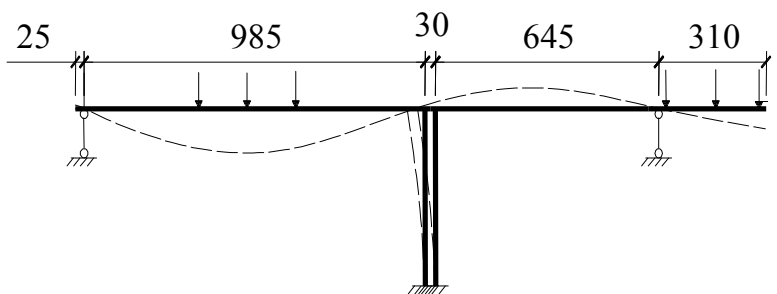

(b)

Fig. 4 Models with or without considering width of bearings: (a) The normal situation without loading; (b) Supposing the actual supporting position moving according to the deflecting line, as shown in the figure under the action of analysis load case [unit: $\mathrm{cm}$ ]

The displacements and moments along the girder calculated from beam_a and beam_b are shown in Fig.5. Maximum values of the positive moment in the longer span and the negative moment in the shorter span are listed in Table 3. The extreme influence of supporting width is only $2.0 \%$ and negligible on the positive moment in the middle of longer span, while its influence on the negative moment at the side supporting position of the shorter span is up to a quite significant value of $11.8 \%$ and not negligible. Therefore the influence of support width should be taken into account in the load bearing capacity assessment. 


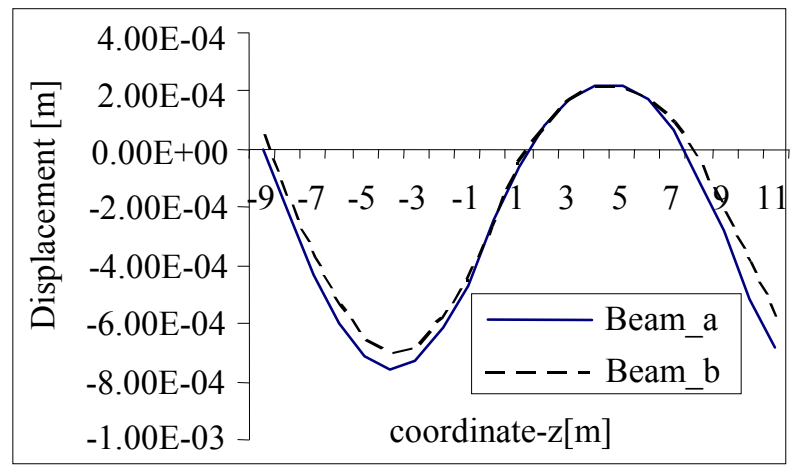

(a) Displacements

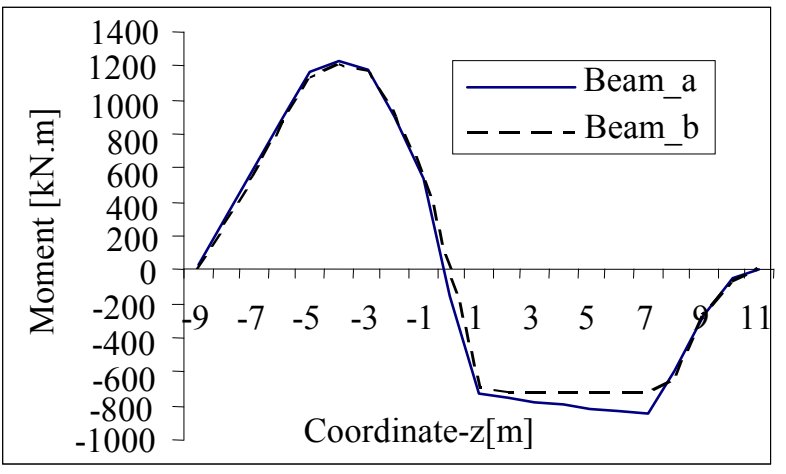

(b) Moments

Fig. 5 Results calculated from model_a and model_b

Table 3 Maximum values of the moment calculated from beam a and beam $\_b$

\begin{tabular}{lcc|ccc}
\hline \multicolumn{2}{c|}{ Positive moment(kN.m) } & \multicolumn{3}{c}{ Negative moment(kN.m) } \\
\hline Beam_a & Beam_b & Diff. percent (\%) & Beam_a & Beam_b & Diff. percent (\%) \\
\hline 1236.20 & 1211.40 & 2.0 & -871.00 & -767.85 & 11.8 \\
\hline
\end{tabular}

Note: Diff.percent $=\frac{\mid \text { Beam_a-Beam_b|}}{\mid \text { Beam_a }{ }_{-} \mid} \times 100 \%$.

Influence of Additional Support. The comparison of calculated results from shell_a and shell_b is shown in Fig. 6(a) - (c). It can be found from these figures that the additional support influences very slightly on the displacements and the stresses of the longer span, while it affects much on the results of the shorter span and the cantilever, especially around the supported area.

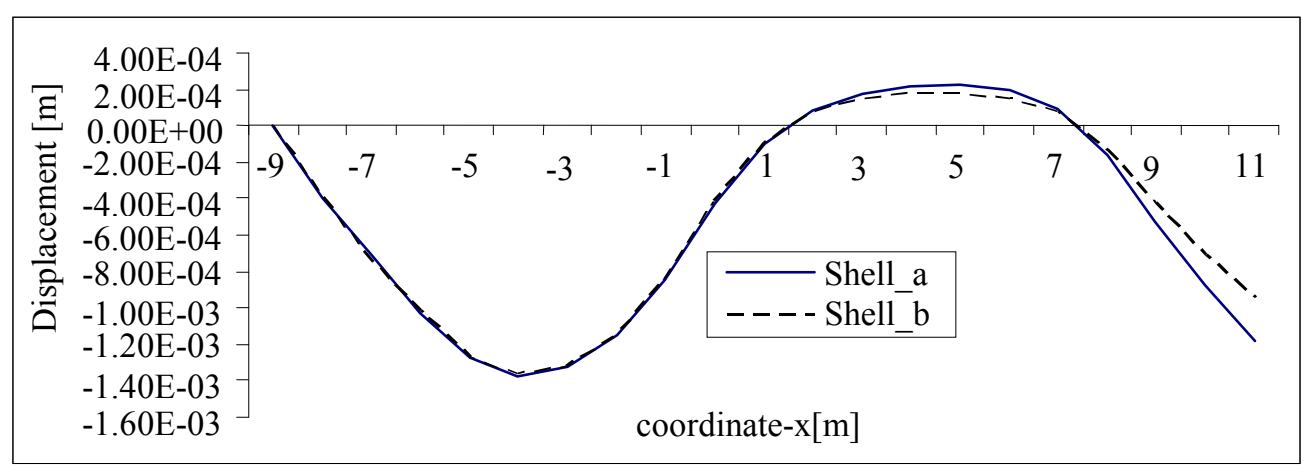

(a) Displacements along the girder

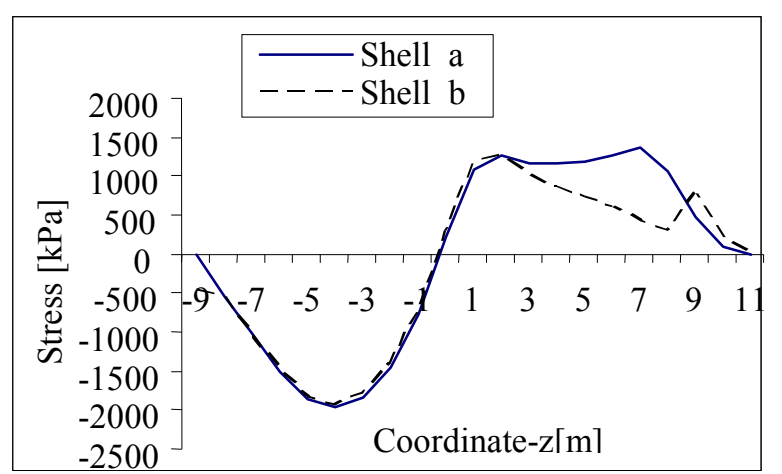

(b) Stresses of the top

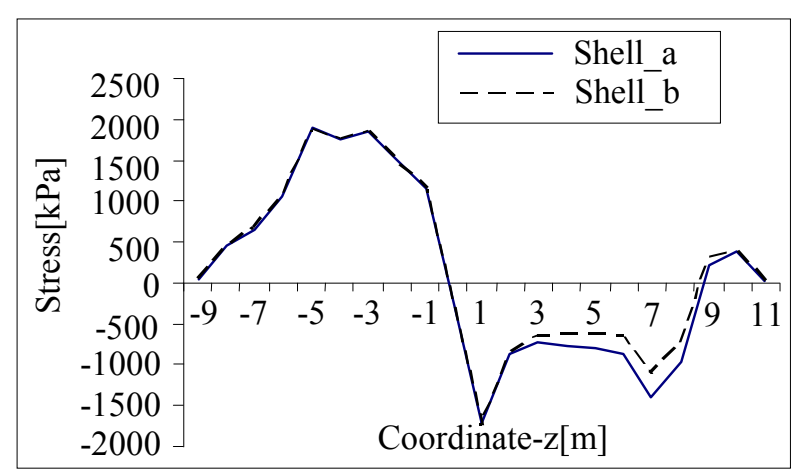

(c) Stresses of the bottom

Fig. 6 Results of shell_a and shell_b with different boundary condition. The displacements along the girder and stresses of the bottom refer to the results of central line of the bottom face, while stresses of the top refer to the results of central line of the flange top face. 
Stress Distribution in the Girder. Fig. 7 shows the transversal distribution of normal stresses in the bottom side of the girder. It can be found that the normal stresses of the cross section are strongly unevenly distributed. The deviation extent of the normal stresses for each cross section can be evaluated by its mean absolute difference value (MADV), as shown in Fig. 8. The deviation of the normal stresses is distinctly, especially around the bearings; and it is much more obvious in model_b and model_d, which implies that the influence of the additional support is very significant.

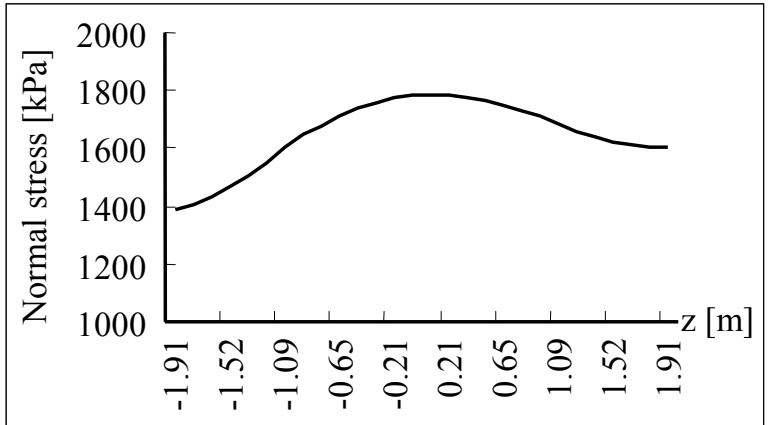

a) Middle of the longer span

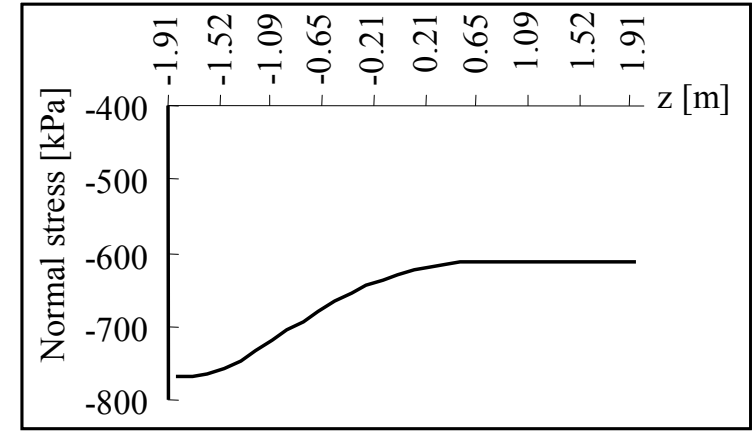

b) Middle of the shorter span

Fig. 7 Transversal distribution of normal stresses in the bottom side of the slab

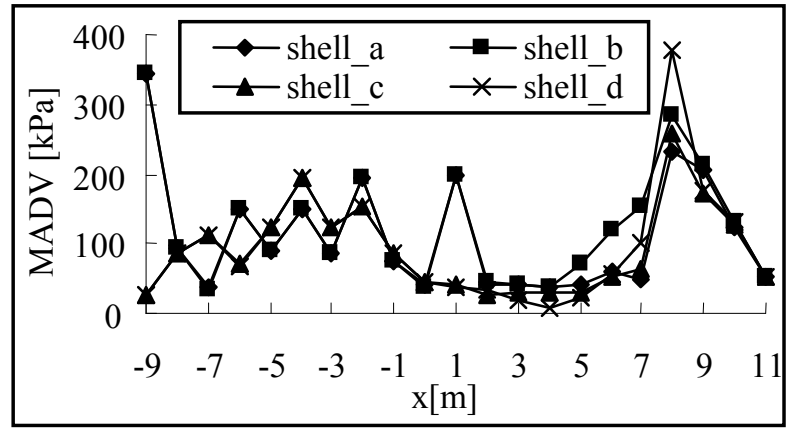

a) Bottom side (bottom of the slab)

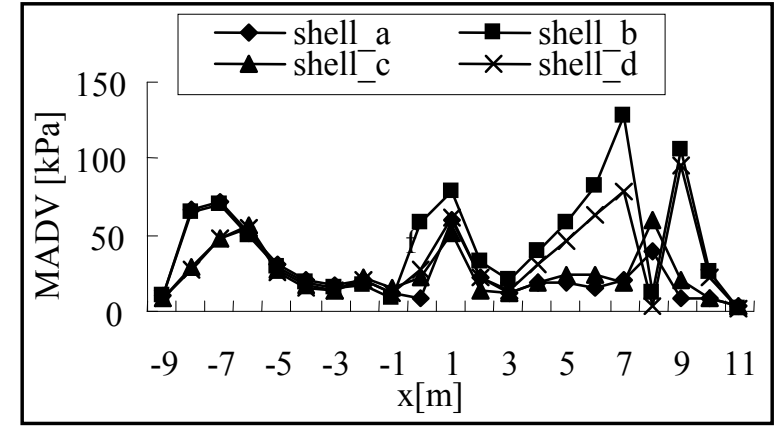

b) top side (top of the flange)

Fig. 8 Mean absolute difference value (MADV) of the normal stresses along transversal direction of each cross-section

\section{Calibration of the Calculations with the Measurements}

To investigate the actual responses of the bridge under the action of train load, some tests were carried out and the displacements and stresses of the girder were measured [2]. The layout of the sensors is shown in Fig. 9. For more details about the field tests see [2]. Some extracted values from calculations and measurements with respect to the analysis load case are listed in Table 4 for comparison.
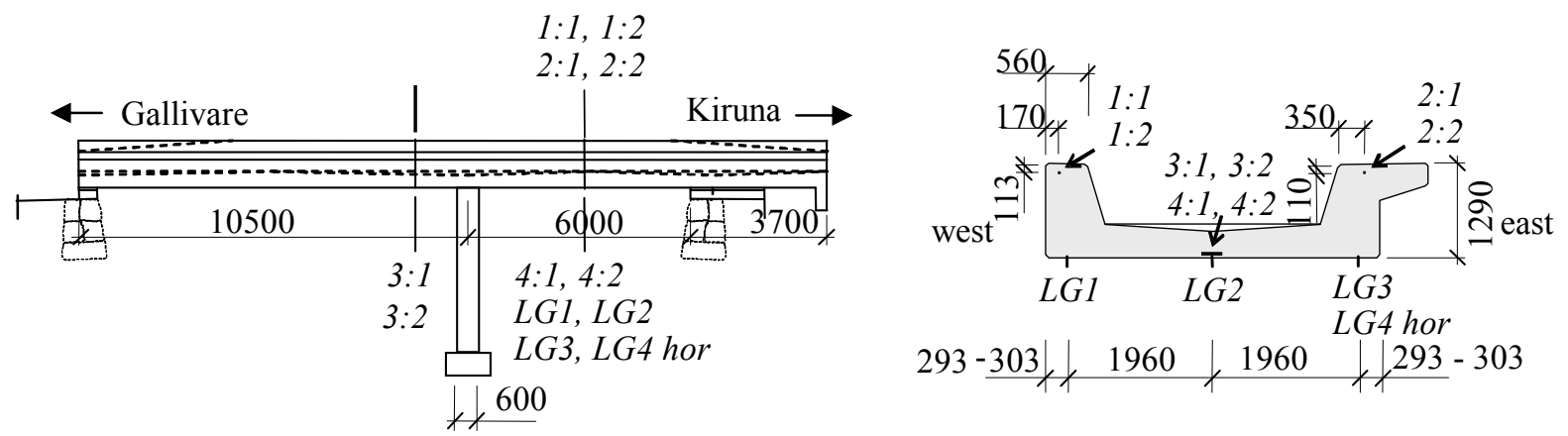

Fig. 9 Layout of the measuring sets: 8 stress gauges and 4 displacement measures (excerpted from [2] with adjusted form) 
Table 4 Comparison of calculations and measurements

\begin{tabular}{|c|c|c|c|c|c|c|c|c|}
\hline & \multicolumn{6}{|c|}{ Calculated results } & \multirow{2}{*}{$\begin{array}{l}\text { Measuredr } \\
\text { esults }\end{array}$} \\
\hline & & Beam_a & Beam_b & Shell_a & Shell_b & Shell_c & Shell_d & \\
\hline \multirow{3}{*}{$\begin{array}{l}\text { Displacement } \\
{[\mathrm{mm}]}\end{array}$} & LG1 & \multirow{3}{*}{+0.23} & \multirow{3}{*}{+0.22} & +0.25 & +0.24 & +0.24 & +0.23 & +0.14 \\
\hline & LG 2 & & & +0.23 & +0.18 & +0.21 & +0.17 & +0.04 \\
\hline & LG 3 & & & +0.25 & +0.20 & +0.24 & +0.19 & +0.09 \\
\hline \multirow{4}{*}{$\operatorname{Strain}[\mu \varepsilon]$} & $1: 11: 2$ & \multirow{2}{*}{+37.5} & \multirow{2}{*}{+34.1} & +40.6 & +39.8 & +36.5 & +35.2 & +37 \\
\hline & $2: 12: 2$ & & & +35.3 & +24.1 & +31.6 & +22.9 & +28 \\
\hline & $3: 13: 2$ & / & / & +8.5 & +7.7 & +8.5 & +7.8 & +8 \\
\hline & $4: 14: 2$ & / & / & -1.1 & +0.5 & -0.3 & +1.3 & +2 \\
\hline
\end{tabular}

It can be concluded from Table 4 that: i) the variation rule of the calculations is in good accordance with that of the measurements, which implies that the accuracy of the analysis is credible; ii) measured strains lie between the calculations of shell_a and shell_c or shell_b and shell_d, so the simulated situations shown in Fig. 4(a)(b) are the two extreme cases for the variation of the span; iii) strains at the upper west side $(1: 1,1: 2)$ are larger than those at the upper east $\operatorname{side}(2: 1,2: 2)$, but the difference values in shell_a and shell_c are much smaller than those of shell_b and shell_d, while the measured difference value lies between them. It indicates that the additional support acts significant influence on the stress distribution in the girder, but not as much as calculated in shell_b and shell_d.

\section{Conclusions}

Based on the FE-analysis and calibration with the measurements, the following conclusions are drawn:

(1)The influences of bearing width and the additional support on the static behavior of the Luossajokk Bridge are significant, so they should be taken into account in the capacity assessment.

(2) More researches are needed to learn the actual exact supporting position and additional supporting length if more accurate stress distribution is required.

(3) Considering the evident spatial effect of stress distribution in the girder, greater safety coefficient of the load response should be adopted in the load bearing capacity assessment.

(4) In the load bearing capacity assessment of small span bridges like the Luossajokk Bridge [3-4], the uncertainty of boundaries and spatial effect of load response should be taken into account.

\section{Acknowledgment}

The authors acknowledge the Swedish Railway Administrator "Banverket" for financing the project.

\section{References}

[1] Ola Enochsson, Arvid Hejll and Martin Nilsson, etc: Bending capacity assessment of the middle section of shorter span of the Luossajokk Bridge by safety index method. Research report, March 2002 (in Swedish)

[2] Ola Enochsson, Arvid Hejll and Martin Nilsson: Measurements of the Luossojokk Bridge in 2002 - 2003. Research report, March 2002 (in Swedish)

[3] James, Gerard: Raising allowable axle loads on railway bridges using simulation and field Data. Licentiate thesis. Department of Structural Engineering, Royal Institute of Technology, 2001.

[4] Paulsson, Björn; Kellner, Håkan and Öström, Lars: Increasing axle loads on railway bridges. Stahlbau, 1998, vol. 67, No. 8, pp 667-671. 